\title{
Strong diameter-dependence of nanowire emission coupled to waveguide modes
}

Dick van Dam', Diego R. Abujetas, José A. Sánchez-Gil, Jos E. M. Haverkort, Erik P. A. M. Bakkers, and Jaime Gómez Rivas'

Citation: Appl. Phys. Lett. 108, 121109 (2016); doi: 10.1063/1.4944848

View online: $h$ ttp://dx.doi.org/10.1063/1.4944848

View Table of Contents: http://aip.scitation.org/toc/apl/108/12

Published by the American Institute of Physics

AIP | Applied Physics Letters

Save your money for your research.

It's now FREE to publish with us -

no page, color or publication charges apply.

If your article has the

potential to shape the future of

applied physics, it BELONGS in

Applied Physics Letters 


\title{
Strong diameter-dependence of nanowire emission coupled to waveguide modes
}

\author{
Dick van Dam, ${ }^{1, a)}$ Diego R. Abujetas, ${ }^{2}$ José A. Sánchez-Gil, ${ }^{2}$ Jos E. M. Haverkort, ${ }^{1}$ \\ Erik P. A. M. Bakkers, ${ }^{1,3}$ and Jaime Gómez Rivas ${ }^{1,4, b)}$ \\ ${ }^{1}$ Department of Applied Physics, Eindhoven University of Technology, P.O. Box 513, 5600 MB Eindhoven, \\ The Netherlands \\ ${ }^{2}$ Instituto de Estructura de la Materia (IEM-CSIC), Consejo Superior de Investigaciones Científicas Serrano, \\ 121, 28006 Madrid, Spain \\ ${ }^{3}$ Kavli Institute of Nanoscience, Delft University of Technology, Lorentzweg 1, 2628 CJ Delft, The Netherlands \\ ${ }^{4}$ Dutch Institute for Fundamental Energy Research DIFFER, P.O. Box 6336, 5600 HH Eindhoven, \\ The Netherlands
}

(Received 12 January 2016; accepted 15 March 2016; published online 24 March 2016)

\begin{abstract}
The emission from nanowires can couple to waveguide modes supported by the nanowire geometry, thus governing the far-field angular pattern. To investigate the geometry-induced coupling of the emission to waveguide modes, we acquire Fourier microscopy images of the photoluminescence of nanowires with diameters ranging from 143 to $208 \mathrm{~nm}$. From the investigated diameter range, we conclude that a few nanometers difference in diameter can abruptly change the coupling of the emission to a specific mode. Moreover, we observe a diameter-dependent width of the Gaussian-shaped angular pattern in the far-field emission. This dependence is understood in terms of interference of the guided modes, which emit at the end facets of the nanowire. Our results are important for the design of quantum emitters, solid state lighting, and photovoltaic devices based on nanowires. (C) 2016 AIP Publishing LLC. [http://dx.doi.org/10.1063/1.4944848]
\end{abstract}

Vertically standing semiconductor nanowires are of interest for the realization of quantum optical devices, ${ }^{1,2}$ lightemitting diodes (LEDs), ${ }^{3}$ and solar cells. ${ }^{4,5}$ For all of these applications, the angle-dependent (or directional) interaction of nanowires and light is of great importance. For instance, quantum emitters require excellent coupling into fiber optics, for which a Gaussian angular emission pattern is advantageous, ${ }^{6}$ while LEDs typically need a narrow beam for efficient illumination. ${ }^{7}$ Furthermore, solar cells require omnidirectional light absorption to trap diffuse light, although unidirectional absorption might be preferential for optimal solar cell efficiency in the radiative limit, ${ }^{8}$ i.e., the re-emission cone of light from solar cells needs to be as narrow as possible in order to match the incident solid angle of solar radiation and thus reduce entropy losses. ${ }^{9-11}$ In all these cases, control over the directional emission and absorption is crucial for the device performance.

Both the directional emission ${ }^{12,13}$ and directional absorption $^{14,15}$ of light in individual semiconductor nanowires have been investigated recently. Indium phosphide (InP) and gallium arsenide (GaAs) have proven to be among the leading materials for quantum emitters ${ }^{16,17}$ and solar cells ${ }^{5,18}$ based on nanowires. For these applications and materials, the approximate optimal diameter for absorbing the solar spectrum has been estimated to be $177-220 \mathrm{~nm}^{17,19}$ The reason for this optimal diameter is the onset of efficient coupling to the fundamental HE11 waveguide mode, ${ }^{20}$ which improves both absorption (for photons with energy just above the material bandgap energy) and guiding/outcoupling (for photons with energy below the material bandgap energy). The first transverse waveguide

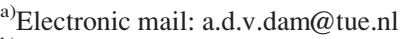

b)Electronic mail: j.gomezrivas@differ.nl
}

modes (TM01 and TE01) have their cut-off diameter close to the optimal diameter, which may influence the directional outcoupling (and absorption) of light, as these modes show a distinctly different directional emission profile. ${ }^{13,21}$ However, the range of diameters close to the onset of the transverse guided modes has not been investigated experimentally.

In this letter, we measure the directional emission from nanowires with eight different diameters in the range from 143 to $208 \mathrm{~nm}$, and conclude that the coupling to waveguide modes is very sensitive to the diameter. The width of the Gaussian angular pattern is found to be diameter-dependent, with a minimum width around a diameter of $164 \mathrm{~nm}$. There is an abrupt change in the emission pattern when the nanowire diameter exceeds $171 \mathrm{~nm}$. These measurements illustrate the relevance of carefully tuning the nanowire diameter with nanometer accuracy to optimize device performance.

Our sample consists of square-symmetric arrays of indium phosphide (InP) nanowires, that have been fabricated by sequential axial vapour-liquid-solid (VLS) growth and radial vapour-solid (VS) growth, as has been described elsewhere. ${ }^{13,22}$ The nanowires from different arrays exhibit different diameters $d$ and share the same length of about $7 \mu \mathrm{m}$. The nanowires are untapered although the top and bottom ends (both $1 \mu \mathrm{m}$ of the length) have a slight tapering. This tapering has no effect on the result, as has been addressed in our previous work. ${ }^{13}$ The nanowires were excited with a $640 \mathrm{~nm}$ diode laser under a $100 \times$ microscope objective with a numerical aperture of 0.95 . A typical photoluminescence spectrum is shown in Figure 1 (red, dashed curve), which is blueshifted with respect to the substrate emission (black, solid curve). The nanowires exhibit band gap emission, which points at a predominantly wurtzite crystal structure, other than the substrate, which has a zincblende crystal structure. We investigate the 


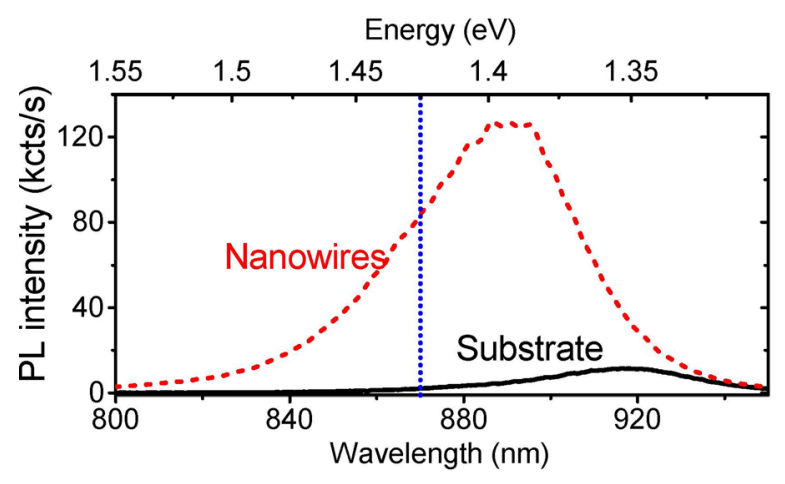

FIG. 1. Typical microphotoluminescence spectrum from the nanowires (red, dashed) and the supporting substrate (black, solid). The blue, dotted line indicates the central wavelength of the band pass filter used in the experiments.

directional emission from the nanowires by Fourier microscopy. This technique, also known as back focal plane imaging, uses the property of an objective lens to project a certain emission direction onto a specific point in the back focal plane. The experimental setup has been described in detail elsewhere. ${ }^{12}$

Figure 2 shows the directional emission patterns from the eight nanowire arrays that we have measured, accompanied by a scanning electron micrograph from one of them (Figure 2(a)). Figure 2(b) explains the measurement geometry and the coordinates of the emission patterns, while Figure 2(c) shows the measured emission patterns. The first row shows the unpolarized emission patterns, while the second (third) row shows the patterns recorded with the polarizer oriented horizontally (vertically) with respect to the images, as indicated by the double arrows on the left side. A gradual narrowing of the emission pattern is visible when increasing the diameter from 143 to $164 \mathrm{~nm}$, as well as an abrupt change of the pattern around $d=171 \mathrm{~nm}$. For larger diameters, we observe, instead of a Gaussian-like pattern, a significantly different pattern with a pronounced dip in the center of the images. This doughnut-like shape is modified by the periodic array of nanowires although the azimuthal emission is not significantly changed. ${ }^{13}$

Since it is known that the emission pattern is mainly determined by waveguide modes, ${ }^{12,13}$ we display the dispersion diagram $\left(k_{z d}\right.$ vs. $\left.\omega d / c\right)$ of the relevant waveguide modes in Figure 3(a). On the bottom horizontal axis, we show the diameters for a frequency fixed to the emission frequency of InP, $\omega / c=k_{0}=2 \pi / 870 \mathrm{~nm}$, and InP refractive index $n_{\text {InP }}=3.43$. We show also the light line for air (in gray), which defines the boundary between guided modes (above the light line) and leaky modes (below the light line). The leaky modes are similar to the guided modes but have a complex propagation constant in the direction along the nanowire (with $\operatorname{Re}\left(k_{z}\right)<\omega / c$ ), which makes them leaky and radiating into the far field. ${ }^{23}$ In the diameter range of the experiment, only the HE11, TM01, and TE01 modes are available. The TM01 (TE01) mode is leaky for diameters below $d=203 \mathrm{~nm}$ (between $d=172 \mathrm{~nm}$ and $d=203 \mathrm{~nm}$ ) and guided for diameters above $d=203 \mathrm{~nm}$. Since the TM and TE modes are polarized, the polarization of the emission provides information about the coupling to these waveguide modes. Therefore, we calculate the polarization anisotropy ratio, defined as $\rho=\left(\bar{I}_{\|}-\bar{I}_{\perp}\right) /\left(\bar{I}_{\|}+\bar{I}_{\perp}\right),{ }^{24,25}$ for the angleintegrated emission of each nanowire. In this equation, $\bar{I}_{\|}$ corresponds to the angle-integrated emission component parallel to the nanowire, whereas $\bar{I}_{\perp}$ is the emission component perpendicular to the nanowire. ${ }^{26} \rho$ is displayed in Figure 3(b), as a function of the nanowire diameter. We see that $\rho$ remains very close to 0 , up to about $d=170 \mathrm{~nm}$, where it abruptly becomes negative to a value of about $-10 \%$. This negative value indicates a larger perpendicularly polarized emission fraction, pointing at a coupling of the emission to the TE01 mode, which becomes available (although leaky) around a diameter of $170 \mathrm{~nm}$. We see no signatures of the TM01 mode, which can be related to the fact that its dispersion is very close to the light line, or to the (mainly) wurtzite crystal structure of the nanowires. Wurtzite material forbids
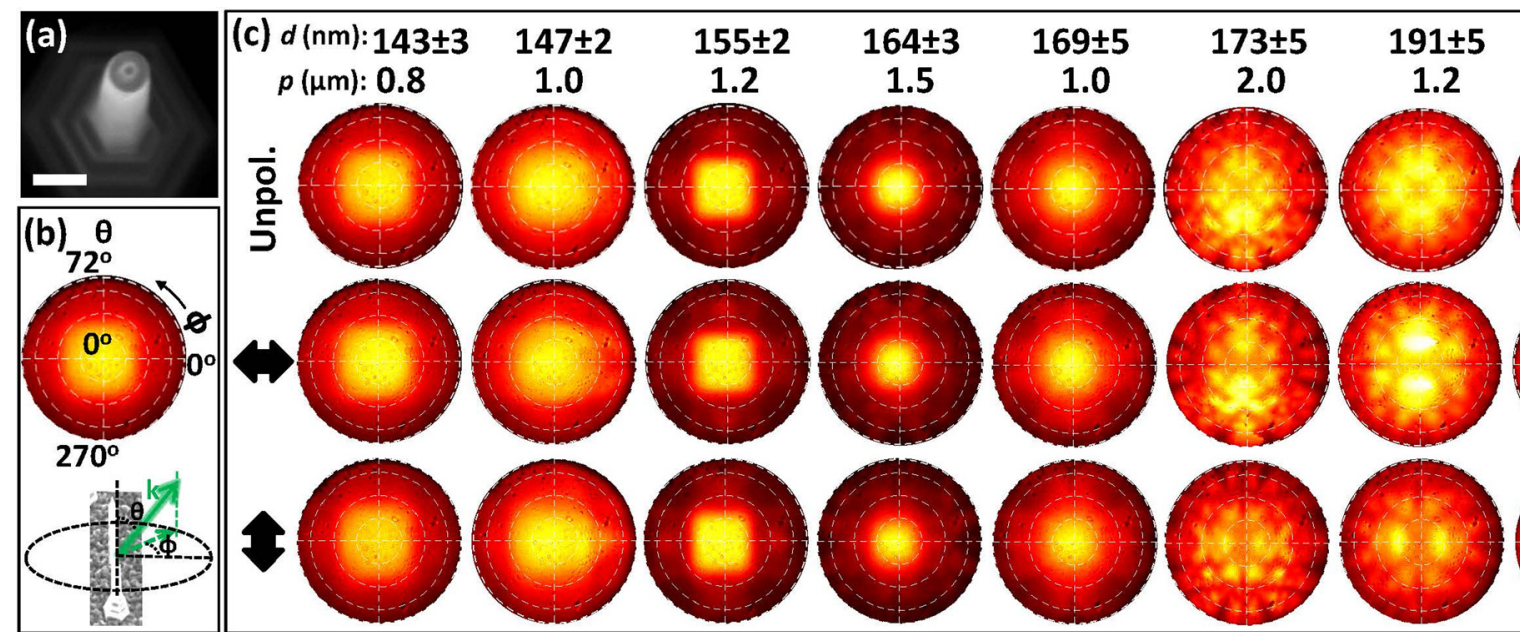

$208 \pm 6$

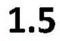

FIG. 2. Polarized directional emission from nanowires. (a) Scanning electron micrograph of one of the investigated nanowires. The scale bar is 200 nm. (b) Example of an emission pattern and definition of the elevation angle $\theta$ (between the long axis of the nanowire and the emission k-vector) and the azimuthal angle $\phi$. (c) Emission patterns of nanowires with different diameters. The first row shows the unpolarized emission, and in the second (third) row, only the emission with polarization horizontal (vertical) with respect to the image is recorded. The uncertainties in the diameter denote wire-to-wire differences and the error in the imaging. $p$ is the period of the nanowire array. 


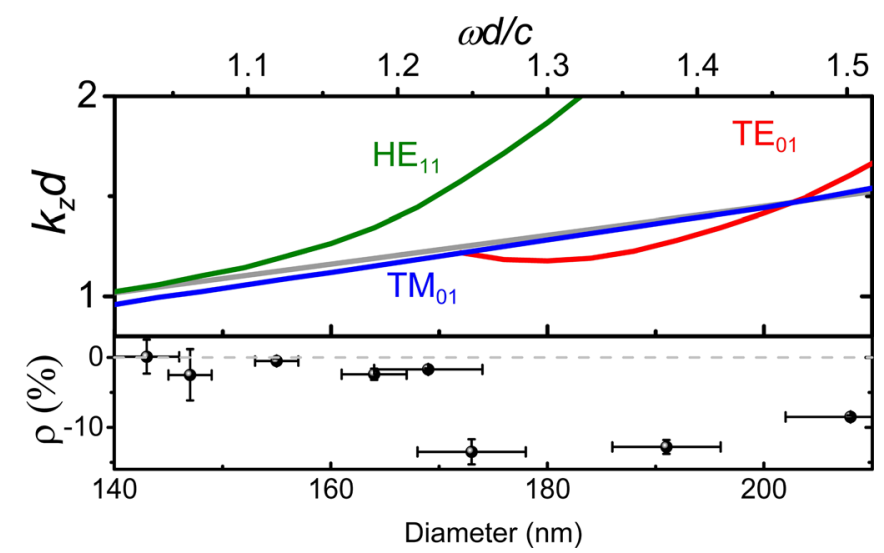

FIG. 3. Waveguide modes in InP nanowires and polarization anisotropy. (a) Representation of the waveguide mode dispersion, i.e., $k_{z} d$ as a function of $\omega d / c$. The gray line is the light line in air. The corresponding diameters for a fixed emission wavelength of $870 \mathrm{~nm}$ ( $\mathrm{InP}$ refractive index of 3.43) are shown at the bottom horizontal axis (shared with b). (b) Polarization anisotropy $\rho=\left(I_{\|}-I_{\perp}\right) /\left(I_{\|}+I_{\perp}\right)$ as a function of the diameter determined from Figure 2(c).

dipole emission oriented parallel to the nanowire, ${ }^{27,28}$ which is needed to couple efficiently to the TM01 mode.

Figure 4(a) displays the profiles of the directional emission patterns along $\phi=0^{\circ}$. We compare this emission to the calculated emission profiles which correspond to the relevant waveguide modes. These calculated emission profiles were determined using an analytical model, ${ }^{29}$ which envisions the nanowire as a one dimensional current in a wire of a finite length $L$ excited by a point dipole at a distance $z_{0}$ from the nanowire center. ${ }^{26} \mathrm{We}$ calculate the emission patterns by fixing the $k_{z}$ 's corresponding to the HE11 and TE01 modes at each given diameter. These $k_{z}$ 's are determined from the dispersion curves shown in Figure 3(a).
For $d<170 \mathrm{~nm}$, only the HE11 mode profiles are calculated, and for $d>170 \mathrm{~nm}$, both the HE11 (dotted) and TE01 (dashed) modes, as well as an average. We conclude that at $d<170 \mathrm{~nm}$, the emission can be explained by the HE11 mode. At $d>170 \mathrm{~nm}$, we see a strong emission at $\theta=0^{\circ}$ as well, which also points at coupling of the emission to the HE11 mode. However, the features at emission angles of $20^{\circ}<\theta<40^{\circ}$ are mainly polarized perpendicular to the nanowire (as can be seen in Figure 2(c), second and third rows), which can only be explained by emission guided by the TE01 mode. Therefore, we conclude that both modes are excited for $d>170 \mathrm{~nm}$. The solid lines in Figure 4(b), which are the average between the profiles of the HE11 and TE01 modes, show good agreement with the measurement.

As mentioned before, we observe a gradual transition among the thinner nanowires $(d<170 \mathrm{~nm})$, although only a single mode (HE11) is excited in this range. To quantify this transition, we display in Figure 5(a) the full-width at half maximum (FWHM) of the angular profiles shown in Figure 4(a). We observe a narrowing of the profile when increasing the diameter, finding a minimum $\theta_{\text {FWHM }}$ of $47^{\circ}$ around $d=164 \mathrm{~nm}$. The profile broadens for $d=169 \mathrm{~nm}$. This broadening cannot be related to coupling to the TE01 mode because this mode is not supported for this diameter. A very similar trend is visible in the calculated $\theta_{\text {FWHM }}$ from the profiles shown in Figure 5(b). To account for slight changes of the nanowire length, we show in this figure the results for nanowire lengths between 6 and $8 \mu \mathrm{m}$. The calculations were based on a 1D model, with only the parallel component of the mode's wave vector $\left(k_{z}\right)$, the source position distribution, and end facet reflectivities as parameters. Of these parameters, only the mode's wave vector changes when modifying $d$ in the relevant regime. Therefore, we conclude that the behaviour of $\theta_{\mathrm{FWHM}}$ is controlled only by (a) Measured

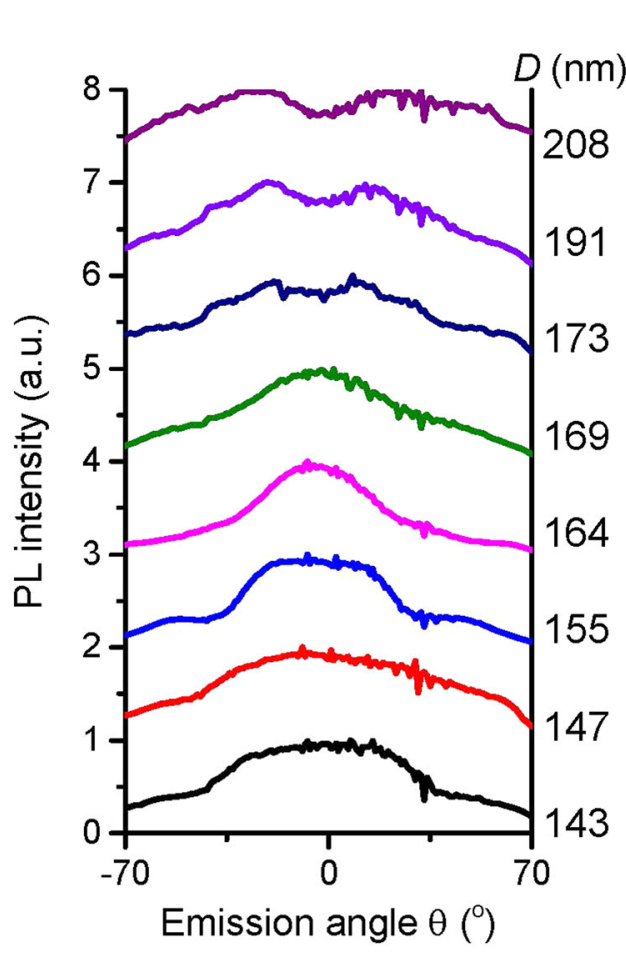

(b) Calculated

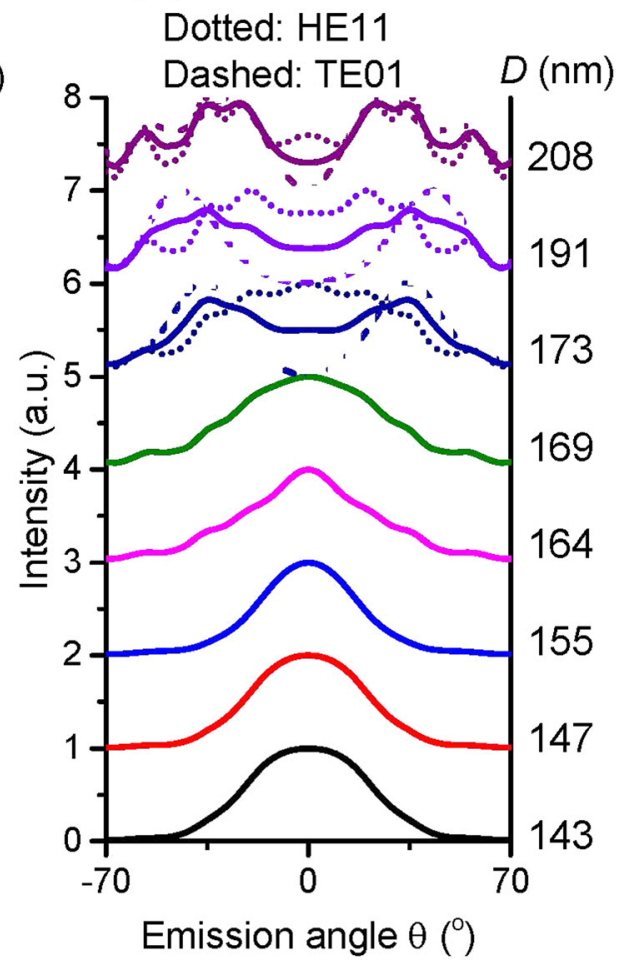

FIG. 4. Stacked directional emission profiles along $\phi=0^{\circ}$. (a) Measured profiles of the normalized directional unpolarized emission profiles (Figure 1(c), first row). (b) Calculated profiles using a 1D analytical model. At diameters smaller than $170 \mathrm{~nm}$, only the HE11 mode is supported. At diameters larger than $170 \mathrm{~nm}$, both HE11 and TE01 modes are shown (the dotted lines represent the HE11 mode calculations, and the dashed lines represent the TE01 mode calculations. The solid line is the average between the two modes). 

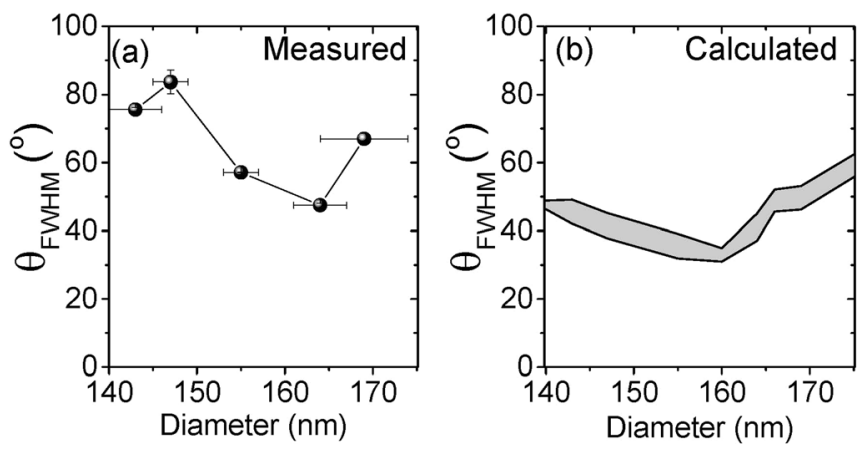

FIG. 5. FWHM of the directional emission. (a) Measured angular full-with at half maximum (FWHM) of the directional emission profiles that are associated with the HE11 mode. (b) Calculated angular FWHM using an analytical model. The shaded area shows the variation for a nanowire length of 6 to $8 \mu \mathrm{m}$.

$k_{z}$, thus by the effective mode wavelength $\lambda_{\text {eff }}$ (since $\left.k_{z}=2 \pi / \lambda_{\text {eff }}\right)$. This parameter determines, together with the nanowire length, the interference pattern of the emission from the end facets of the nanowire into the far field, and therefore also the width of the Gaussian-like distribution. Although there is a reasonably good qualitative agreement between the measurements and the analytical model, there are significant quantitative discrepancies. These discrepancies can be attributed to the simplicity of the $1 \mathrm{D}$ model and the presence of the substrate in the measurements, which introduces an additional reflection at the bottom interface. Additionally, the increased $\theta_{\text {FWHM }}$ in the thinnest measured nanowires might be caused by scattering due to the surrounding nanowires because the period is smaller for these nanowire arrays.

In conclusion, we have shown that a change in nanowire diameter of only a few nanometers may induce an abrupt change in the emission pattern and polarization. This change is related to the onset of the TE01 leaky mode, to which the emission can couple. Also, we have found that the width of the emission pattern of the HE11 mode is strongly diameterdependent, with a minimum width around $d=164 \mathrm{~nm}$. This is caused by the interference of light which couples out at the nanowires end facets. This work provides important guidelines for the design for quantum emitters, LEDs, and photovoltaic devices based on semiconductor nanowires.

The authors acknowledge Alessandro Cavalli for SEM imaging. This research was supported by the Dutch technology foundation STW, which is part of the "Netherlands Organisation for Scientific Research (NWO)," and partially funded by the Dutch Ministry of Economic Affairs. This work was also part of the research program of the "Foundation for Fundamental Research on Matter (FOM)," which is financially supported by NWO. J.A.S.G. acknowledges the Spanish Ministerio de Economía y Competitividad for financial support through the Grant Nos. NANOPLAS+ (FIS2012-31070) and LENSBEAM (FIS2015-69295-C3-2-P).

${ }^{1}$ N. Panev, A. I. Persson, N. Sköld, and L. Samuelson, Appl. Phys. Lett. 83, 2238 (2003).

${ }^{2}$ I. Friedler, C. Sauvan, J. P. Hugonin, P. Lalanne, J. Claudon, and J.-M. Gérard, Opt. Express 17, 2095 (2009).

${ }^{3}$ K. Haraguchi, T. Katsuyama, K. Hiruma, and K. Ogawa, Appl. Phys. Lett. 60, 745 (1992).

${ }^{4}$ K. Peng, Y. Xu, Y. Wu, Y. Yan, S. T. Lee, and J. Zhu, Small 1, 1062 (2005).

${ }^{5}$ J. Wallentin, N. Anttu, D. Asoli, M. Huffman, I. Åberg, M. H. Magnusson, G. Siefer, P. Fuss-Kailuweit, F. Dimroth, B. Witzigmann, H. Q. Xu, L. Samuelson, K. Deppert, and M. T. Borgström, Science 339, 1057 (2013).

${ }^{6}$ G. Bulgarini, M. E. Reimer, M. Bouwes Bavinck, K. D. Jöns, D. Dalacu, P. J. Poole, E. P. A. M. Bakkers, and V. Zwiller, Nano Lett. 14, 4102 (2014).

${ }^{7}$ A. Köck, E. Gornik, M. Hauser, and W. Beinstingl, Appl. Phys. Lett. 57, 2327 (1990)

${ }^{8}$ Y. Xu, T. Gong, and J. N. Munday, Sci. Rep. 5, 13536 (2015).

${ }^{9}$ G. L. Araújo and A. Martí, Sol. Energy Mater. Sol. Cells 33, 213 (1994).

${ }^{10}$ E. D. Kosten, J. H. Atwater, J. Parsons, A. Polman, and H. A. Atwater, Light: Sci. Appl. 2, e45 (2013).

${ }^{11}$ U. Rau, U. W. Paetzold, and T. Kirchartz, Phys. Rev. B 90, 035211 (2014).

${ }^{12}$ G. Grzela, R. Paniagua-Domínguez, T. Barten, Y. Fontana, J. A. SánchezGil, and J. Gómez Rivas, Nano Lett. 12, 5481 (2012).

${ }^{13}$ D. van Dam, D. R. Abujetas, R. Paniagua-Domínguez, J. A. Sánchez-Gil, E. P. A. M. Bakkers, J. E. M. Haverkort, and J. Gómez Rivas, Nano Lett. 15, 4557 (2015).

${ }^{14}$ L. Cao, J. S. White, J.-S. Park, J. A. Schuller, B. M. Clemens, and M. L. Brongersma, Nat. Mater. 8, 643 (2009).

${ }^{15}$ G. Grzela, R. Paniagua-Domínguez, T. Barten, D. van Dam, J. A. Sánchez-Gil, and J. Gómez Rivas, Nano Lett. 14, 3227 (2014).

${ }^{16}$ J. Claudon, J. Bleuse, N. S. Malik, M. Bazin, P. Jaffrennou, N. Gregersen, C. Sauvan, P. Lalanne, and J.-M. Gérard, Nat. Photon. 4, 174-177 (2010).

${ }^{17}$ M. E. Reimer, G. Bulgarini, N. Akopian, M. Hocevar, M. Bouwes Bavinck, M. A. Verheijen, E. P. A. M. Bakkers, L. P. Kouwenhoven, and V. Zwiller, Nat. Commun. 3, 737 (2012).

${ }^{18}$ I. Åberg, G. Vescovi, D. Asoli, U. Naseem, J. P. Gilboy, C. Sundvall, A. Dahlgren, K. E. Svensson, N. Anttu, M. T. Björk, and L. Samuelson, IEEE J. Photovoltaics 6, 185 (2016).

${ }^{19}$ N. Anttu and H. Q. Xu, Opt. Express 21, A558 (2013).

${ }^{20}$ K. Seo, M. Wober, P. Steinvurzel, E. Schonbrun, Y. Dan, T. Ellenbogen, and K. B. Crozier, Nano Lett. 11, 1851 (2011).

${ }^{21}$ A. V. Maslov and C. Z. Ning, Opt. Lett. 29, 572 (2004).

${ }^{22}$ G. Bulgarini, M. E. Reimer, T. Zehender, M. Hocevar, E. P. A. M. Bakkers, L. P. Kouwenhoven, and V. Zwiller, Appl. Phys. Lett. 100, 121106 (2012).

${ }^{23}$ A. W. Snyder and J. D. Love, Optical Waveguide Theory (Chapman and Hall, 1983), pp. 488-501.

${ }^{24}$ J. Wang, M. S. Gudiksen, X. Duan, Y. Cui, and C. M. Lieber, Science 293, 1455 (2001).

${ }^{25}$ H. E. Ruda and A. Shik, J. Appl. Phys. 100, 024314 (2006).

${ }^{26}$ See supplementary material at http://dx.doi.org/10.1063/1.4944848 for a schematic description of the polarization-dependent analysis and further details about the model.

${ }^{27}$ J. Birman, Phys. Rev. 114, 1490 (1959).

${ }^{28}$ C. Wilhelm, A. Larrue, X. Dai, D. Migas, and C. Soci, Nanoscale 4, 1446 (2012).

${ }^{29}$ R. Paniagua-Domínguez, G. Grzela, J. Gómez Rivas, and J. A. SánchezGil, Nanoscale 5, 10582 (2013). 